$12-13-2011$

\title{
Cerebrospinal fluid rhinorrhea: An institutional perspective from Pakistan
}

\author{
Muhammad Zubair Tahir \\ Aga Khan University, zubair.tahir@aku.edu \\ Muhammad Babar Khan \\ Aga Khan University \\ Muhammad Umair Bashir \\ Aga Khan University \\ Shabbir Akhtar \\ Aga Khan University, shabbir.akhtar@aku.edu \\ Muhammad Ehsan Bari \\ Aga Khan University, ehsan.bari@aku.edu
}

Follow this and additional works at: https://ecommons.aku.edu/pakistan_fhs_mc_surg_neurosurg

Part of the Neurology Commons, Otolaryngology Commons, and the Surgery Commons

\section{Recommended Citation}

Tahir, M., Khan, M., Bashir, M., Akhtar, S., Bari, M. (2011). Cerebrospinal fluid rhinorrhea: An institutional perspective from Pakistan. Surgical Neurology International, 2, 174.

Available at: https://ecommons.aku.edu/pakistan_fhs_mc_surg_neurosurg/14 


\title{
Original Article
}

\section{Cerebrospinal fluid rhinorrhea: An institutional perspective from Pakistan}

\author{
Muhammad Zubair Tahir, Muhammad Babar Khan, Muhammad Umair Bashir, Shabbir Akhtar ${ }^{1}$, \\ Ehsan Bari
}

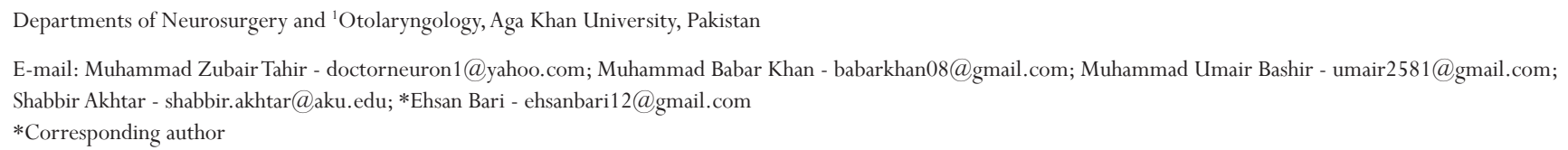

\begin{abstract}
Background: The management of cerebrospinal fluid (CSF) rhinorrhea has evolved over the last two decades. We present here a review of our 11-year data on CSF rhinorrhea and its management at a tertiary care hospital in a developing country, with particular reference to the diagnosis, surgical management and outcome of the disease.
\end{abstract}

Methods: The medical charts of all patients with a diagnosis of CSF rhinorrhea over an 11-year period were reviewed. The etiology of CSF rhinorrhea was classified into three categories: spontaneous, iatrogenic and traumatic. All the patients were divided into three categories based on the type of management as conservative, intracranial and transnasal endoscopic groups.

Results: A total of 43 patients fulfilled our inclusion criteria and were included in the final analysis. Eleven of the 43 patients were managed conservatively, while 22 underwent intracranial repairs; 10 patients had transnasal endoscopic repairs. The primary success rate for the transnasal approach was $70 \%$ compared to $86 \%$ for the intracranial repair. Blood loss, special care unit (SCU) stay and total cost were found to be significantly less in the transnasal endoscopic group. Computed tomography (CT) cisternography was found to have the highest sensitivity and specificity. Further, no postoperative complications were found in the transnasal endoscopic group, while five patients from the intracranial group developed various complications.

Conclusions: We conclude that the transnasal endoscopic approach has comparable success rates with the intracranial approach and significantly lower morbidity.

Key Words: Cerebrospinal fluid rhinorrhea/diagnosis, cerebrospinal fluid rhinorrhea/etiology, cerebrospinal fluid rhinorrhea/surgery, endoscopy, retrospective studies, treatment outcome

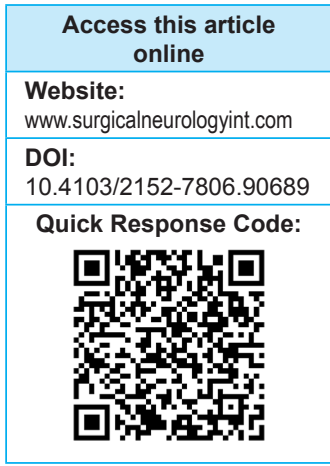

\section{INTRODUCTION}

Cerebrospinal fluid (CSF) rhinorrhea occurs when there is a communication between the subarachnoid space and the sinonasal mucosa due to meningeal and osseous defects in the cranial base, leading to discharge of CSF 
from the nose. The majority of cases are traumatic in etiology, mostly caused by accidental head trauma or iatrogenic injury. ${ }^{[12]}$ However, CSF leaks may also be secondary to high intracranial pressure, tumors, erosive diseases, congenital skull base defects, and some may be spontaneous with no specific etiology. ${ }^{[5,20]}$ Most cases of persistent CSF rhinorrhea need a definitive intervention, with the risk of meningitis in untreated patients reported to be about $10 \%$ annually and up to $40 \%$ in long-term follow-up. ${ }^{[10,16,18]}$

Numerous methods have been employed for the management of CSF rhinorrhea, ranging from conservative therapies such as bed rest to complicated surgical repairs. Surgical management of CSF leak itself encompasses a variety of approaches, including craniotomy-based intracranial techniques as well as minimally invasive endoscopic repairs. The first successful intracranial repair of a CSF leak was reported by Dandy in $1926 .^{[1]}$ Since then, intracranial repair by craniotomy has resulted in cure for $70-80 \%$ of patients. ${ }^{[8,19]}$ The traditional intracranial approach has the advantage of direct visualization of the dural tear and also the ability to manage complex bone fractures and use pericranial flaps in repair. However, such an invasive approach that also involves brain retraction has the associated morbidity of anosmia, memory deficits, hemorrhage, cerebral edema and osteomyelitis of the bone flap. ${ }^{[15]}$ The introduction of the extracranial repair through a naso-orbital incision by Dohlman in $1948^{[2]}$ and the endoscopic approach to a leak repair in $1981^{[23]}$ were attempts to overcome the relative morbidity of the earlier intracranial approaches. Since the first extracranial attempts for CSF leak repair, a number of case series and studies have highlighted the reduced morbidity and efficacy of the endoscopic techniques. ${ }^{[7,18]}$ The reduction in complications along with the avoidance of skin incisions has currently made the transnasal endoscopic repair the procedure of choice for CSF leak repair at most centers worldwide.

We present here a review of our 11-year data on CSF rhinorrhea and its management at a tertiary care hospital in a developing country, with particular reference to the diagnosis, surgical management and outcome of the disease. At the end, the intracranial repairs have been compared with endoscopic transnasal endoscopic repairs, with the objective of defining the most appropriate current management of CSF rhinorrhea.

\section{MATERIALS AND METHODS}

The hospital database and clinical coding system encompassing an 11-year period from January 2000 to May 2011 were examined for diagnosis of CSF rhinorrhea. Hospital charts, electronic records and radiologic studies for these patients were reviewed using a standardized proforma. The data collected included demographic data, associated co-morbidities, leak sites, etiology, presenting complaints, type of repair, lumbar drain use, fluorescein use, perioperative management, recurrence and length of follow-up.

The etiology of CSF rhinorrhea was classified into three categories - spontaneous, iatrogenic and traumatic which included leaks secondary to blunt and penetrating injuries. The leak sites were divided into the following categories: sphenoid sinus, ethmoid sinus including both anterior and posterior ethmoid cells, cribriform plate and frontal sinus. The diagnostic techniques used to confirm the presence and site of CSF rhinorrhea included computed tomography (CT), magnetic resonance imaging (MRI), CT cisternography and intraoperative fluorescein use.

All the patients were divided into three categories based on the type of management as conservative, intracranial and transnasal endoscopic groups. The various types of graft materials used in the operative procedures along with other perioperative management (lumbar drains, nasal packs) were also reviewed. The two operative groups were compared with regards to duration of surgery, blood loss, hospital stay, total cost, recurrence and complications. The primary success rate for a particular group was defined by the number of patients with complete resolution of symptoms after the initial surgery. The secondary success rate for each group was based on the number of patients treated successfully after a second attempt at repair of CSF rhinorrhea.

The Gaussian distribution assumption was tested using the Kolmogorov-Smirnov test on all the continuous variables analyzed for the two operative groups. The variables (blood loss, duration of surgery and total cost) that succeeded in passing the normality test were compared using the Student's $t$-test, while the variables (SCU stay and total hospital stay) that failed the test of normality were compared by the Mann-Whitney Wilcoxon test for independent samples. Statistical analysis was performed using SPSS software (SPSS Inc., Chicago, IL, USA); a $P$-value of less than 0.05 was taken to be statistically significant for all comparisons.

The follow-up duration of all the patients was determined, with some patients contacted via telephone to determine their follow-up status. All patients with incomplete/missing records were excluded from the study.

\section{RESULTS}

A total of 43 patients fulfilled our inclusion criteria and were included in the final analysis. Of the 43 patients, 17 were males and 26 were females. Patient age ranged from 3 to 74 years, with a mean age at repair of 40.6 years. Sixteen patients were admitted from the emergency (ER), while 27 were elective hospital admissions. The 
presenting complaints included watery rhinorrhea in 16 (37\%) patients, a combination of headache/fever/altered sensorium/nausea and vomiting in $22(51.2 \%)$ and a host of other complaints including epistaxis, anosmia and visual complaints in $5(11.6 \%)$ patients. Fifteen of the 43 patients were later diagnosed with meningitis after a CSF culture. The follow-up data of all 43 patients were reviewed, with a mean follow-up of 15 months (range: 3-60 months)

The CSF leaks were divided into three categories based on etiology: spontaneous, traumatic (accidents/falls, blunt and penetrating trauma) and iatrogenic [Figure 1]. Iatrogenic leaks were secondary to functional endoscopic sinus surgery (FESS) $(\mathrm{n}=2)$, pituitary adenoma resections $(\mathrm{n}=2)$ and repair of frontal sinus fractures $(\mathrm{n}=3)$.

The initial diagnosis of CSF rhinorrhea was made on the basis of history and examination findings. The betatransferrin assay, used commonly in other countries, is not available in Pakistan, and hence could not be used in the diagnostic workup. Following a preliminary diagnosis, 27 patients were further investigated with a plain CT and 16 patients with a CT cisternogram, while an MRI was done for 18 patients. Intraoperative leak localization was aided with fluorescein in 10 patients and the leak site was accurately determined in 9 of them. The patients were given $0.1 \mathrm{ml}$ of $10 \%$ intrathecal fluorescein via a lumbar drain that was inserted after intubation for CSF leak repair. All leak sites identified intraoperatively were compared with the defects determined on imaging for the respective patients. This information was used to calculate the sensitivity and specificity of the various diagnostic modalities. CT cisternography was found to have the highest sensitivity and specificity [Table 1].

Out of the 43 patients, CSF defects were clearly identified in 40 patients based on investigations and/or intraoperative findings. The most common location of

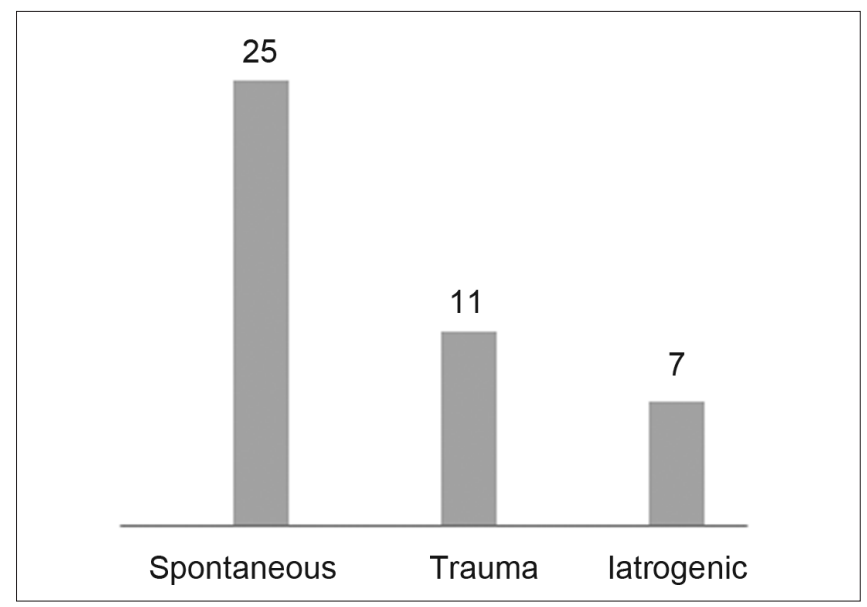

Figure I: Etiology of leaks the defect was the cribriform plate in 16 patients $(40 \%)$ [Figure 2].

A total of 32 patients underwent surgery, while 11 patients were managed conservatively.

\section{Conservative management}

Eleven of the 43 patients were managed conservatively, with a favorable outcome in 6 of the patients. The etiology in this group included six spontaneous leaks, two trauma-related, and three iatrogenic injuries. The routine management involved acetazolamide, laxatives, prophylactic antibiotics and other measures such as bed rest, avoidance of sneezing, etc. A lumbar drain was inserted in three patients with a successful outcome.

Five patients did not respond to conservative management; four of these subsequently underwent a successful intracranial repair while one patient was managed with endoscopic transnasal repair. The leak etiology was spontaneous in four of the five patients and iatrogenic in one. A review of the imaging of these patients revealed that three of them had defects in the cribriform plate, one had an ethmoidal defect, while another patient had a frontal sinus defect.

\section{Operative management}

Over the last 11 years, a total of 32 patients underwent an operative repair. The type of repair, as was evident during chart review, reflected the specific expertise available at that time, with the earlier cases being mostly intracranial.

\section{Table 1: Imaging sensitivity and specificity}

\begin{tabular}{lccc}
\hline Scan & $\begin{array}{c}\text { Numbers } \\
\text { performed }\end{array}$ & $\begin{array}{c}\text { Sensitivity* } \\
(\%)\end{array}$ & $\begin{array}{c}\text { Specificity* } \\
(\%)\end{array}$ \\
\hline CT & 27 & 48.2 & 45 \\
CT cisternogram & 16 & 100 & 93.8 \\
MRI & 18 & 61.1 & 66.6 \\
\hline
\end{tabular}

*Defects identified on imaging were compared with leak sites identified intraoperatively

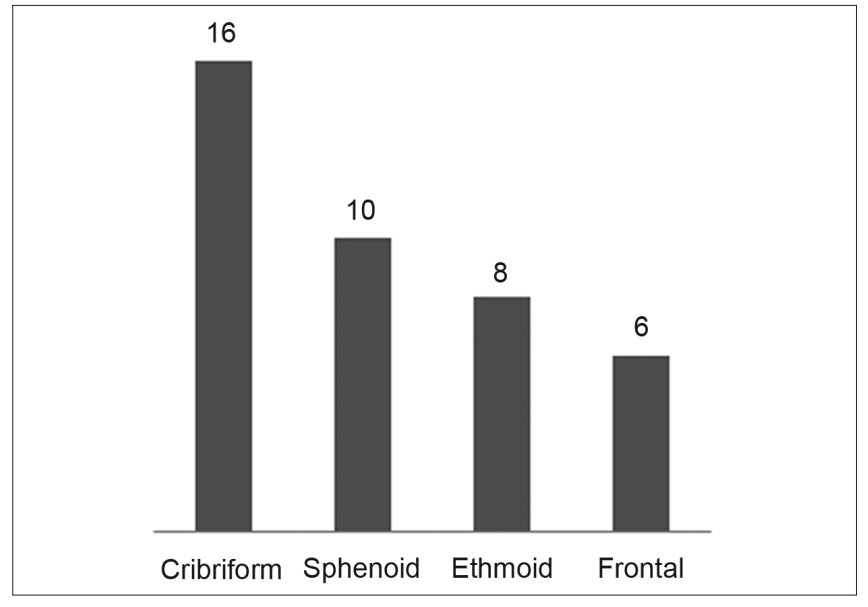

Figure 2: Site of defect 
A total of 22 patients underwent intracranial procedures to repair their CSF defects. Nineteen of the 22 patients were successfully treated after the primary surgery. CSF rhinorrhea persisted in one patient after the initial surgery and recurrence was seen in another two patients at 24 months and 8 months, respectively. Two of the three patients were cured of CSF leak after a second intracranial surgery, while a second intracranial repair failed in the third patient; he subsequently underwent a successful transnasal endoscopic procedure. A lumbar drain was inserted postoperatively in 11 patients for an average of 2 days.

Ten of the 32 patients underwent a transnasal endoscopic procedure. Intraoperative fluorescein was successfully used to aid in the localization of the defect in nine cases. However, CSF rhinorrhea persisted in 2 of the 10 patients immediately after surgery and recurred in 1 patient at 6 months. All the three cases, however, were successfully managed via a transnasal endoscopic approach in the second attempt. Postoperatively, nasal packs were used in eight patients for an average of 1.6 days while lumbar drains were routinely inserted in five patients for an average of 2.5 days. No infectious complications were observed in the patients at follow-up.

The differences between the two procedures in terms of blood loss, duration of surgery, SCU stay, total hospital stay and cost are presented in Table 2. A comparison between the two groups using the Student's $t$-test or Mann-Whitney Wilcoxon test, as appropriate, revealed that blood loss, SCU stay and total cost were significantly less in the transnasal endoscopic group $(P$ values of $0.03,0.03$ and 0.04 , respectively). Follow-up data were reviewed in all 43 patients, with an average duration of 15 months. A review of the follow-up records of the transnasal endoscopic group did not reveal any significant complications. However, one patient from the intracranial group developed a superficial wound infection, three patients complained of persistent headaches and two patients reported a loss of smell at a follow-up visit.

\section{DISCUSSION}

This is a comprehensive retrospective review of CSF

Table 2: Transnasal endoscopic versus intracranial

\begin{tabular}{lccc}
\hline & $\begin{array}{c}\text { Transnasal } \\
\text { endoscopic }\end{array}$ & Intracranial & $\boldsymbol{P}$ \\
\hline Variables & Mean \pm SD & Mean \pm SD & \\
Blood loss & $116 \pm 72$ & $431 \pm 94$ & 0.03 \\
Duration of surgery & $131 \pm 20$ & $176 \pm 17$ & 0.12 \\
SCU stay & $0.9 \pm 0.2$ & $4.1 \pm 1.2$ & $0.03^{*}$ \\
Total hospital stay & $7.9 \pm 1.3$ & $13.3 \pm 2.2$ & $0.06^{*}$ \\
Total cost & $1800 \pm 235$ & $2970 \pm 220$ & 0.04 \\
\hline
\end{tabular}

*Mann-Whitney Wilcoxon rhinorrhea and its management over 11 years at the Aga Khan University Hospital (AKUH), which is a tertiary care center in a developing country. Although other centers in more developed countries have consistently and widely written about their experiences, our study is the first of its kind from this region and its results mirror the facilities and expertise available at our center for CSF rhinorrhea management over the last decade. In this context, most of the earlier cases in this series were managed transcranially irrespective of cause and location of defect, as this was the only intervention that was available. However, more recently, the transnasal endoscopic approach has become common due to the availability of trained surgeons. Some of the results of this study are also better interpreted in the context of the learning curve phenomenon, particularly those related to the endoscopic approach.

Incidence and epidemiological studies about CSF rhinorrhea are lacking in Pakistan. It is generally considered a rare entity with a low index of suspicion. The average duration of symptoms in our case series was 8 weeks before a final diagnosis was made, mostly by an otolaryngologist or a neurosurgeon. Although a CSF rhinorrhea is reported to be intermittent or occult in $20-30 \%$ cases $^{[18,22]}$ in literature, the low index of suspicion among general practitioners in Pakistan coupled with the absence of a screening test (beta-transferrin) in the country might also account for such a long interval before CSF rhinorrhea was diagnosed in our group of patients. Such a delayed diagnosis also explains the high incidence of meningitis in our study $(\mathrm{n}=15)$, with 3 of the 15 patients having a documented history of recurrent meningitis.

In our series, CSF leaks mostly involved adults in the range of 30-50 years, with a distinct majority of women in the spontaneous leak group $(\mathrm{n}=20,43.5 \%)$. The most common site involved for spontaneous and traumatic leaks in our series was the cribriform plate $(\mathrm{n}=11$ and 4 , respectively). Common sites reported in literature include the sphenoid and cribriform for spontaneous leaks, the frontoethmoid and cribriform for traumatic ones and the ethmoid for iatrogenic leaks. ${ }^{[1]}$ Iatrogenic leak sites in our study involved the ethmoid $(\mathrm{n}=2)$, frontal sinus ( $\mathrm{n}$ $=3$ ) and sphenoid $(\mathrm{n}=1)$. All the frontal sinus leaks were found to be secondary to repair of traumatic frontal sinus fractures.

Over the last decade, a number of imaging techniques have yielded varying results in the detection and localization of CSF leaks. Both CT and MRI have been advocated as noninvasive tests to adequately localize the site of CSF leak. ${ }^{[13]}$ However, CT imaging detects the fluid poorly, and multiple thin sections with a high resolution may give rise to a large number of false positives, ${ }^{[22]}$ leading to unwarranted interventions if the 
leak site is not confirmed with another modality. MRI cisternography, on the other hand, offers poor spatial and bony resolution. ${ }^{[6]}$ Both of these modalities combined, however, have a higher sensitivity and specificity. ${ }^{[14,21]}$ However, in a resource-constrained environment like ours, where high-resolution CT is not widely available and dual modality analysis is not cost-effective, CT cisternography offers an acceptable tradeoff between accurate detection, localization and planning of intervention. ${ }^{[4,13]}$ This modality becomes all the more appealing because an active CSF leak on a CT cisternogram is the only means of actually diagnosing CSF rhinorrhea in Pakistan as beta-transferrin or beta-trace protein assays are not available. In our study, CT cisternography was able to identify a defect in all the patients it was performed, with its specificity approaching 94\% when compared to intraoperative findings. Therefore, we suggest CT cisternography with a non-ionic contrast as the primary diagnostic modality in a setting like ours.

This study also intends to audit and compare the two types of approaches for the repair of CSF leaks at AKUH over the last decade. The intracranial approach was the only intervention available at our center for a number of years until the transnasal endoscopic technique was introduced. In this context, the success rate after the primary attempt for intracranial cases was $86 \%$ compared to $70 \%$ for the cases using the transnasal endoscopic approach. The recurrence rate for the two approaches was almost comparable (9\% for the intracranial and $10 \%$ for transnasal endoscopic cases). Overall, however, the transnasal endoscopic approach had a higher success rate $(\mathrm{n}=100 \%)$ after repeat attempts were considered [Table 3]. Success rates for the intracranial approach reported in literature are in the range of $70-80 \%$ and 86-100\% for the transnasal endoscopic approach. $[3,7,11,15,18]$ The morbidity of the intracranial approach, however, was significantly higher compared to the transnasal endoscopic approach, with a higher incidence of wound infection, severe headache and anosmia in the intracranial group.

A further comparison revealed a significant difference in blood loss, SCU stay and total cost between the two groups. Although it was hypothesized that there would be a considerable difference in the mean duration of surgery too, our analysis showed an insignificant difference, possibly secondary to longer surgery times for the earlier transnasal endoscopic cases. Intraoperative fluorescein was

Table 3: Primary and secondary success rates

\begin{tabular}{lcc}
\hline Management & $\begin{array}{c}\text { Success on } 1^{\text {st }} \\
\text { attempt }(\%)\end{array}$ & $\begin{array}{c}\text { Success on } 2^{\text {nd }} \\
\text { attempt }(\%)\end{array}$ \\
\hline Transnasal endoscopic & $7 / 10(70)$ & $3 / 3(100)$ \\
Intracranial & $19 / 22(86)$ & $2 / 3(66.7)$ \\
Conservative & $6 / 11$ & - \\
\hline
\end{tabular}

used in 10 transnasal endoscopic cases, with successful localization in 9 of them. Based on this experience, we believe that intraoperative fluorescein should be used for accurate localization. The most appropriate time to inject fluorescein, however, remains surgeon and institution dependent. Fluorescein injected after intubation eliminates the morbidity of a preoperative lumbar puncture and improves patient comfort. Hence, it is our institutional practice to inject fluorescein with a lumbar drain inserted after intubation. Also, no adverse effects were observed with intrathecal fluorescein $(0.1 \mathrm{ml}$ of $10 \%$ ) in our group of patients.

Several different types of grafting material have been used with good results in the treatment of CSF rhinorrhea. In their meta-analysis, Hegazay et al. found no statistically significant difference among different grafting techniques and materials. ${ }^{[7,17]}$ A variety of grafts were also used in our series, including fat, fascia, cartilage and bone with fibrin glue in various combinations. No significant difference between the success rates and the use of various grafts was found. Although we tried to analyze the data regarding the use of lumbar drains and nasal packs, our sample size was too small to come up with appreciable conclusions. However, Hegazy et al. advocated the use of lumbar drains for 3-5 days postoperatively with idiopathic, traumatic and recurrent leaks and with large meningocele herniation. ${ }^{[7]}$ Also, lumbar drains are recommended in the repair of frontal and sphenoid sinus defects. ${ }^{[9]}$

To conclude, our study is an institutional review of CSF rhinorrhea and its management over the last decade. CSF rhinorrhea is not a common disease entity, but has significant morbidity if the diagnosis is delayed. The traditional intracranial approach may still have a role in the management of complex defects, but the endoscopic technique is clearly superior with high overall success and low rate of complications in the hands of a trained surgeon. Hence, the transnasal endoscopic approach should be considered as the first option for repair of all uncomplicated CSF leaks.

\section{REFERENCES}

I. Dandy WD. Pneumocephalus(intracranial pneumatocele or aerocele). Arch Surg 1926; 12:949-82.

2. Dohlman G. Spontaneous cerebrospinal fluid rhinorrhea. Acta Otolaryngol Suppl (Stockh) 1948;67:20-3.

3. Giddings NA, Brackmann DE. Surgical treatment of difficult cerebrospinal fluid otorhinorrhea.Am J Otol 1994; 15:781-4.

4. Gulcek SY, Erdogan A, Toprak U, Ortac AA, Pasaoglu E. [Evaluation of rhinorrhea by computed tomography cisternography]. Tani Girisim Radyol 2003;9:327-32.

5. Har-El G.What is "spontaneous" cerebrospinal fluid rhinorrhea? Classification of cerebrospinal fluid leaks. Ann Otol Rhinol Laryngol 1999;108:323-6.

6. Hegarty SE, Millar JS. MRI in the localization of CSF fistulae: Is it of any value? Clin Radiol 1997;52:768-70.

7. Hegazy HM, Carrau RL, Snyderman CH, Kassam A, Zweig J. Transnasal endoscopic repair of cerebrospinal fluid rhinorrhea: $A$ meta-analysis. Laryngoscope 2000; I I0: I 166-72. 
8. Lanza DC, O'Brien DA, Kennedy DW. Endoscopic repair of cerebrospinal fluid fistulae and encephaloceles. Laryngoscope 1996;106:1 I 19-25.

9. Lee TJ, Huang CC, Chuang CC, Huang SF. Transnasal endoscopic repair of cerebrospinal fluid rhinorrhea and skull base defect: Ten-year experience. Laryngoscope 2004; I 14:1475-8I.

10. Lewin W. Cerebrospinal fluid rhinorrhea in nonmissile head injuries. Clin Neurosurg 1964;12:237-52.

II. Lindstrom DR, Toohill RJ, LoehrITA, Smith TL. Management of cerebrospinal fluid rhinorrhea:The Medical College ofWisconsin experience. Laryngoscope 2004; I 14:969-74.

12. Lopatin AS, Kapitanov DN, Potapov AA. Endonasal endoscopic repair of spontaneous cerebrospinal fluid leaks. Arch Otolaryngol Head Neck Surg 2003; 129:859-63.

13. Lund VJ, Savy L, Lloyd G, Howard D. Optimum imaging and diagnosis of cerebrospinal fluid rhinorrhoea. J Laryngol Otol 2000; I 14:988-92.

14. Mostafa BE, Khafagi A. Combined HRCT and MRI in the detection of CSF rhinorrhea. Skull Base 2004; I4:157-62; discussion 162.

15. Paludetti G, Sergi B, Rigante M, Campioni P, Galli J. New techniques and technology to repair cerebrospinal fluid rhinorrhea. Acta Otorhinolaryngol Ital 2004;24:130-6.
16. Park Jl, Strelzow VV, Friedman WH. Current management of cerebrospinal fluid rhinorrhea. Laryngoscope 1983;93:1294-300.

17. Prickett KK, Wise SK, Delgaudio JM. Choice of graft material and postoperative healing in endoscopic repair of cerebrospinal fluid leak. Arch Otolaryngol Head Neck Surg 20 I I; I37:457-6I.

18. Sanderson JD, Kountakis SE, McMains KC. Endoscopic management of cerebrospinal fluid leaks. Facial Plast Surg 2009;25:29-37.

19. Schick B, Ibing R, Brors D, DrafW. Long-term study of endonasal duraplasty and review of the literature. Ann Otol Rhinol Laryngol 200 I; I 10:142-7.

20. Schlosser RJ, BolgerWE. Nasal cerebrospinal fluid leaks.J Otolaryngol 2002;3 I Suppl I:S28-37.

21. Shetty PG, Shroff MM, Sahani DV, Kirtane MV. Evaluation of high-resolution $C T$ and MR cisternography in the diagnosis of cerebrospinal fluid fistula. AJNR Am J Neuroradiol 1998; 19:633-9.

22. Stone JA, Castillo M, Neelon B, Mukherji SK. Evaluation of CSF leaks: highresolution $\mathrm{CT}$ compared with contrast-enhanced $\mathrm{CT}$ and radionuclide cisternography. AJNR Am J Neuroradiol 1999;20:706-I2

23. Wigand ME. [Transnasal, endoscopical surgery for chronic sinusitis. III. Endonasal ethmoidectomy (author's transl)]. HNO I981;29:287-93. 